\title{
Focus point gauge mediation in product group unification
}

\author{
Felix Brümmer $^{\mathrm{a}, *}$, Masahiro Ibe ${ }^{\mathrm{b}, \mathrm{c}}$, Tsutomu T. Yanagida ${ }^{\mathrm{b}}$ \\ a Deutsches Elektronen-Synchrotron DESY, D-22603 Hamburg, Germany \\ b Kavli IPMU, TODIAS, University of Tokyo, Kashiwa 277-8583, Japan \\ c ICRR, University of Tokyo, Kashiwa 277-8582, Japan
}

\section{A R T I C L E I N F O}

\section{Article history:}

Received 30 July 2013

Accepted 6 September 2013

Available online 11 September 2013

Editor: J. Hisano

\begin{abstract}
A B S T R A C T
In certain models of gauge-mediated supersymmetry breaking with messenger fields in incomplete GUT multiplets, the radiative corrections to the Higgs potential cancel out during renormalization group running. This allows for relatively heavy superpartners and for a $125 \mathrm{GeV}$ Higgs while the fine-tuning remains modest. In this Letter, we show that such gauge mediation models with "focus point" behaviour can be naturally embedded into a model of $\mathrm{SU}(5) \times \mathrm{U}(3)$ product group unification.
\end{abstract}

(c) 2013 Elsevier B.V. All rights reserved.

\section{Introduction}

If low-energy supersymmetry is realized in nature, the LHC results of the last two years point towards a rather high superpartner mass scale, perhaps in the range of several TeV. However, obtaining an electroweak symmetry breaking scale which is an order of magnitude or more below the superpartner mass scale requires significant fine-tuning. This is the well-known little hierarchy problem of supersymmetry.

Models with non-unified gaugino masses have recently been argued to alleviate the supersymmetric fine-tuning problem in the MSSM [1-7]. For suitable "focus point" ratios of gaugino masses, the radiative corrections to the Higgs potential cancel out during renormalization group running, yielding an electroweak scale which is much smaller than the typical scale of soft SUSY-breaking masses.

High-scale gauge mediation with messenger fields in incomplete GUT multiplets can naturally realize suitable non-universal soft mass ratios [3]. In terms of the messenger indices $N_{2}$ and $N_{3}$ for pairs of fundamental $\mathrm{SU}(2)_{\mathrm{L}}$ and $\mathrm{SU}(3)_{\mathrm{C}}$ messenger multiplets, the favourable models tend to have a ratio around $N_{2}: N_{3} \approx 5: 2$ (if the mediation scale is close to the GUT scale, and if $\tan \beta$ is large). Models with such an exotic field content may be obtained from higher-dimensional orbifold GUTs, or from related heterotic string constructions [4]. In the present Letter we are proposing an example within the more conventional setting of four-dimensional field theory.

\footnotetext{
* Corresponding author.

1 This is similar in spirit to the original focus point SUSY scenario [8-10], where instead the scalar soft mass contributions to the electroweak scale cancel during the renormalization group evolution.
}

Of course any successful GUT model needs to somehow accommodate incomplete GUT multiplets, in order to solve the doublettriplet splitting problem in the Higgs sector. Models of product group unification (PGU) [11-13] achieve this by extending the unified gauge group to, for instance, $\mathrm{SU}(5) \times \mathrm{SU}(3)_{\mathrm{H}} \times \mathrm{U}(1)_{\mathrm{H}}$. Below the GUT scale, colour $\mathrm{SU}(3)_{C}$ is obtained as the diagonal subgroup of $\mathrm{SU}(3)_{\mathrm{H}}$ and the Georgi-Glashow embedded $\mathrm{SU}(3) \subset \mathrm{SU}(5)$, and hypercharge is similarly a linear combination of $U(1)_{H}$ and $U(1) \subset$ SU(5). In such models the doublet-triplet splitting problem is easily solved, and also additional incomplete GUT multiplets can be accommodated straightforwardly.

In PGU models the gauge couplings need not unify. In fact, the theory will become non-perturbative immediately above the GUT scale unless the gauge couplings are prevented from unifying. In order to allow for a cutoff scale which is not at $M_{\mathrm{GUT}}$ but significantly higher, one needs to ensure that $g_{2}$ is the largest among the Standard Model gauge couplings at the GUT scale. This is most easily achieved by adding some additional pairs of $\mathrm{SU}(2)_{\mathrm{L}}$ doublets with intermediate-scale masses.

Intriguingly, PGU and focus point gauge mediation are seen to complement each other. On the one hand, for a focus point-like cancellation in gauge mediation, the model needs to contain significantly more weak doublet messengers than colour triplet messengers. On the other hand, in order to maximize the cutoff scale in PGU one needs to deflect the renormalization group running of $g_{2}$ relative to the other couplings by adding extra vector-like states, again with significantly more weak doublets than colour triplets.

In the present Letter we exploit this observation, by constructing a PGU model with a number of incomplete GUT multiplets with masses below the GUT scale. These will act as gauge mediation messengers, inducing non-unified soft term ratios favourable for naturalness, while at the same time ensuring that the theory remains valid perturbatively at energies above $M_{\mathrm{GUT}}$. 
Our model predicts the lightest Higgs mass to be compatible with the recent LHC discovery, thanks to large radiative corrections from multi-TeV soft terms, and evades the LHC limits on squark and gluino masses. The gauge couplings will become non-perturbative at a cutoff scale $M_{*}$, which is about an order of magnitude larger than $M_{\mathrm{GUT}}$ (but still below the Planck scale).

Given that the present LHC bounds on gluino and squark masses are around $1.5 \mathrm{TeV}$, large parts of the remaining MSSM parameter space are fine-tuned to the level of a permille or worse when taking all soft terms as independent. However, one should bear in mind that the degree of fine-tuning actually depends on the high-scale model in which the soft terms are generated. It is therefore important to search for models in which the fine-tuning is reduced. This can be achieved in models which predict suitable relations between the soft terms, thus lowering the sensitivity of the resulting electroweak scale to the actual fundamental parameters.

In our model, characterized by the messenger indices $N_{2}=17$ and $N_{3}=7$, this is precisely what happens. With this messenger field content, the predicted ratio of gaugino masses is such that the electroweak scale is no longer very sensitive to the actual soft mass scale. Therefore, despite the soft masses being of the order of a few $\mathrm{TeV}$, the fine-tuning is modest compared to generic MSSM models with similarly heavy superpartners. The residual fine-tuning is of the order of a percent.

\section{Field content and evolution of couplings}

We start by briefly reviewing the main properties of the $\mathrm{SU}(5) \times \mathrm{U}(3)$ PGU model; for more details see e.g. [11-13]. Consider a supersymmetric GUT with gauge group $\mathrm{SU}(5) \times \mathrm{SU}(3)_{\mathrm{H}} \times$ $\mathrm{U}(1)_{\mathrm{H}}$. There are three generations of Standard Model matter fields in the $\mathbf{1 0} \oplus \overline{\mathbf{5}}$ and a pair of Higgs fields $H, \bar{H}$ in the $\mathbf{5} \oplus \overline{\mathbf{5}}$ of $\mathrm{SU}(5)$, all of which are uncharged under $\mathrm{SU}(3)_{\mathrm{H}} \times \mathrm{U}(1)_{\mathrm{H}} . \mathrm{Bi}-$ fundamental fields transforming as $Y=(\mathbf{5}, \overline{\mathbf{3}})$ and $\bar{Y}=(\overline{\mathbf{5}}, \mathbf{3})$ under $\mathrm{SU}(5) \times \mathrm{SU}(3)_{\mathrm{H}}$ acquire vacuum expectation values at the GUT breaking scale $M_{\mathrm{GUT}}$, thus breaking $\mathrm{SU}(5) \times \mathrm{SU}(3)_{\mathrm{H}} \times \mathrm{U}(1)_{\mathrm{H}} \rightarrow$ $\mathrm{SU}(3)_{\mathrm{C}} \times \mathrm{SU}(2)_{\mathrm{L}} \times \mathrm{U}(1)_{\mathrm{Y}}$. Here the colour $\mathrm{SU}(3)_{\mathrm{C}}$ emerges as the diagonal subgroup of $\mathrm{SU}(3)_{\mathrm{H}}$ and the Georgi-Glashow embedded $\mathrm{SU}(3) \subset \mathrm{SU}(5)$. Likewise, the hypercharge $\mathrm{U}(1)_{Y}$ is a linear combination of $\mathrm{U}(1)_{\mathrm{H}}$ and the usual hypercharge generator. Adding a pair $T, \bar{T}$ of Higgs triplet partners in the $\mathbf{3} \oplus \overline{\mathbf{3}}$ of $\mathrm{SU}(3)_{\mathrm{H}}$, the superpotential terms

$W=H \bar{T} \bar{Y}+\bar{H} T Y$

give GUT-scale masses to the triplet components of $H$ and $\bar{H}$. Take the hypercharge generator to be

$Y=T^{24}+Q$

where $Q$ is the $\mathrm{U}(1)_{\mathrm{H}}$ charge, and $T^{24}=\sqrt{\frac{3}{5}} \operatorname{diag}\left(\frac{1}{3}, \frac{1}{3}, \frac{1}{3},-\frac{1}{2},-\frac{1}{2}\right)$. The requirement of leaving hypercharge unbroken then fixes the GUT-normalized $\mathrm{U}(1)_{\mathrm{H}}$ charge of $Y$ to be $Q[Y]=-\frac{1}{3}$.

The model also contains a singlet $S$ (which is needed to give a VEV to $Y$ and $\bar{Y}$ ) and an $\mathrm{SU}(3)_{\mathrm{H}}$ adjoint $X$ (which is needed to give masses to the $\mathrm{SU}(3)_{C}$ octet contained in $\left.Y \bar{Y}\right)$. The particle content is summarized in Table 1 . The superpotential is

$$
\begin{aligned}
W= & H \bar{T} \bar{Y}+\bar{H} T Y+S\left(Y \bar{Y}+T \bar{T}-v^{2}\right)+Y X \bar{Y}+T X \bar{T} \\
& + \text { (MSSM Yukawa couplings). }
\end{aligned}
$$

These are all the renormalizable terms allowed by a certain discrete $R$-symmetry, which also serves to forbid dangerous dimension-5 operators.
Table 1

Field content of a minimal model with $S U(5) \times U(3)$ product group unification.

\begin{tabular}{llll}
\hline Field & $\mathrm{SU}(5)$ & $\mathrm{SU}(3)_{\mathrm{H}}$ & $\mathrm{U}(1)_{\mathrm{H}}$ \\
\hline $3 \times \mathbf{1 0}$ & $\mathbf{1 0}$ & $\mathbf{1}$ & 0 \\
$3 \times \overline{\mathbf{5}}$ & $\overline{\mathbf{5}}$ & $\mathbf{1}$ & 0 \\
$H$ & $\mathbf{5}$ & $\mathbf{1}$ & 0 \\
$\bar{H}$ & $\overline{\mathbf{5}}$ & $\mathbf{1}$ & 0 \\
$T$ & $\mathbf{1}$ & $\mathbf{3}$ & $1 / 3$ \\
$\bar{T}$ & $\mathbf{1}$ & $\overline{\mathbf{3}}$ & $-1 / 3$ \\
$Y$ & $\mathbf{3}$ & $-1 / 3$ \\
$\bar{Y}$ & $\mathbf{5}$ & $\mathbf{3}$ & $1 / 3$ \\
$S$ & $\overline{\mathbf{5}}$ & $\mathbf{1}$ & 0 \\
$X$ & $\mathbf{1}$ & $\mathbf{8}$ & 0 \\
\hline
\end{tabular}

Table 2

Messenger fields and their charges.

\begin{tabular}{llll}
\hline Field & $\mathrm{SU}(5)$ & $\mathrm{SU}(3)_{\mathrm{H}}$ & $\mathrm{U}(1)_{\mathrm{H}}$ \\
\hline$N_{5} \times \Phi$ & $\mathbf{5}$ & $\mathbf{1}$ & 0 \\
$N_{5} \times \bar{\Phi}$ & $\overline{\mathbf{5}}$ & $\mathbf{1}$ & 0 \\
$N_{3 \mathrm{H}} \times \Psi$ & $\mathbf{1}$ & $\mathbf{3}$ & $1 / 3$ \\
$N_{3 \mathrm{H}} \times \bar{\Psi}$ & $\mathbf{1}$ & $\overline{\mathbf{3}}$ & $-1 / 3$ \\
\hline
\end{tabular}

To extend this to a model of messenger gauge mediation, we add $N_{5}$ pairs of messengers $\Phi_{I}, \bar{\Phi}_{I}$ in the $\mathbf{5} \oplus \overline{\mathbf{5}}$ of $\operatorname{SU}(5)$, along with $N_{3 \mathrm{H}}$ pairs of additional fields $\Psi_{i}, \bar{\Psi}_{i}$ in the $\mathbf{3} \oplus \overline{\mathbf{3}}$ of $\mathrm{SU}(3)_{\mathrm{H}}$, where $N_{5}>N_{3 \mathrm{H}}$. The $\mathrm{U}(1)_{\mathrm{H}}$ charge assignments are arbitrary so far; we take $Q\left[\Phi_{I}\right]=0$ and $Q\left[\Psi_{i}\right]=\frac{1}{3}$ for simplicity, to match the charges of $H$ and $T$. The superpotential operators

$W=\lambda_{I j} \Phi_{I} \bar{\Psi}_{j} \bar{Y}+\lambda_{I j}^{\prime} \bar{\Phi}_{I} \Psi_{j} Y$

will decouple $N_{3 \mathrm{H}}$ triplets at the scale $M_{\mathrm{GUT}}$, leaving $N_{2} \equiv N_{5}$ doublet pairs and $N_{3} \equiv N_{5}-N_{3 H}$ triplet pairs massless. If $Z=M+F \theta^{2}$ is a SUSY-breaking spurion, the coupling

$W=\kappa_{I J} Z \Phi_{I} \bar{\Phi}_{J}$

will eventually give supersymmetric masses $M$ to all remaining messengers. We assume that $M \ll M_{\mathrm{GUT}}$, such that the GUT-scale massive triplets can be neglected for SUSY breaking mediation, and $F \ll M^{2}$ (a possible dynamical origin of $M$ and $F$ is sketched later in Section 4). This defines a simple model of messenger gauge mediation, with the somewhat unusual property that the light messenger fields do not come in complete GUT multiplets. Table 2 summarizes the messenger field content.

Below the GUT-breaking scale, the messenger couplings become

$W=\sum_{i=1}^{N_{2}} \kappa_{2 i} Z \Phi_{2 i} \bar{\Phi}_{2 i}+\sum_{a=1}^{N_{3}} \kappa_{3 a} Z \Phi_{3 a} \bar{\Phi}_{3 a}$,

where $\Phi_{2 i}$ and $\Phi_{3 a}$ denote the remaining $\operatorname{SU}(2)_{\mathrm{L}}$ doublet and $\mathrm{SU}(3)_{C}$ triplet messengers. Here, we have diagonalized the Yukawa interactions (i.e. the mass matrix of the remaining messenger fields) at leading order without loss of generality. It should be noted that the gauge-mediated MSSM soft masses are independent of the Yukawa couplings $\kappa_{2,3}$ at leading order, since we are assuming that the messengers couple to a single spurion and contributions from the GUT-scale messengers are suppressed.

We can now investigate the evolution of the gauge couplings, using one-loop running and step-function decoupling for a rough estimate. We need to consider three distinct regimes. Below $M$, the field content is that of the MSSM, and the couplings run as usual. Between $M$ and $M_{\mathrm{GUT}}$, the one-loop $\beta$ function coefficients are

$b_{1}=\frac{3}{5}\left(11+N_{2}+\frac{2}{3} N_{3}\right), \quad b_{2}=1+N_{2}, \quad b_{3}=-3+N_{3}$. 
Finally, above $M_{\mathrm{GUT}}$ the $\beta$ function coefficients are

$b_{1 \mathrm{H}}=\frac{3}{5}\left(4+\frac{2}{3} N_{3 \mathrm{H}}\right)=\frac{12}{5}+\frac{2}{5}\left(N_{2}-N_{3}\right)$,

$b_{3 \mathrm{H}}=N_{3 \mathrm{H}}=N_{2}-N_{3}$,

$b_{5}=N_{5}-5=N_{2}-5$.

Furthermore, at $M_{\mathrm{GUT}}$ the couplings satisfy the tree-level matching conditions

$\frac{1}{\alpha_{1}}=\frac{1}{\alpha_{1 \mathrm{H}}}+\frac{1}{\alpha_{5}}, \quad \frac{1}{\alpha_{2}}=\frac{1}{\alpha_{5}}, \quad \frac{1}{\alpha_{3}}=\frac{1}{\alpha_{3 \mathrm{H}}}+\frac{1}{\alpha_{5}}$.

From Eq. (9) it is evident that a unified gauge coupling, $\alpha_{1}=\alpha_{2}=$ $\alpha_{3}$ at $M_{\mathrm{GUT}}$, would correspond to strongly coupled $\mathrm{SU}(3)_{\mathrm{H}}$ and $\mathrm{U}(1)_{\mathrm{H}}$ groups at the GUT-breaking scale. Conversely, if gauge coupling unification is sacrificed by allowing for nonzero $N_{5}$ and $N_{3 H}$, Eqs. (7), (8) and (9) can be used to estimate the scale at which the theory becomes strongly coupled in the UV.

\section{A concrete model}

When including a large number of charged fields, it is difficult to construct a model which remains perturbative all the way to the Planck scale. However, in our model a somewhat lower cutoff scale $M_{*}<M_{\text {Planck }}$ is actually preferable for a number of reasons. First, a cutoff scale around $M_{*}=10^{17} \mathrm{GeV}$ would be of the right order to explain the lack of $m_{s}-m_{\mu}$ unification, as the Yukawa couplings are corrected by higher-dimensional operators such as $W=\mathbf{1 0 5} \bar{H} \bar{Y} Y /\left(M_{*}\right)^{2}{ }^{2}$ And second, a sub-Planckian cutoff allows for a solution of the Polonyi problem (which is generally a concern for high-scale gauge mediation, as it is for gravity mediation; see e.g. [14]) using the mechanism of adiabatic suppression [15].

We therefore choose the cutoff scale to be $M_{*}=10^{17} \mathrm{GeV}$. Moreover, we take the GUT-breaking scale to be $M_{\mathrm{GUT}}=10^{16} \mathrm{GeV}$, and the messenger scale to be $M=10^{14} \mathrm{GeV}$-note that a large separation between the messenger scale and the GUT-breaking scale is preferred, because we will neglect any contributions to the soft terms from GUT-scale massive triplet messengers. ${ }^{3}$

In our model $\tan \beta$ is large, and $\mu$ is smaller than the soft SUSY-breaking terms, because $\mu$ and $B_{\mu}$ are only generated by subdominant gravity-mediated effects. In terms of the running parameters at the soft mass scale $M_{\mathrm{IR}}$, large $\tan \beta$ implies

$m_{Z}^{2}=-\left.2\left(|\mu|^{2}+m_{H_{u}}^{2}\right)\right|_{M_{\mathrm{IR}}}$,

and thus to obtain a realistic electroweak scale, the contributions to $m_{H_{u}}^{2}$ from the various soft terms have to approximately cancel out in the renormalization group evolution. This will at most happen for a few select choices of messenger indices $N_{2}$ and $N_{3}$. Numerically solving the two-loop renormalization group equations between $M_{\mathrm{UV}}=10^{14} \mathrm{GeV}$ and $M_{\mathrm{IR}}=5 \times 10^{3} \mathrm{GeV}$ yields

$$
\begin{aligned}
\left.m_{H_{u}}^{2}\right|_{M_{\mathrm{IR}}}= & \left(-0.79 M_{3}^{2}+0.20 M_{2}^{2}-0.01 M_{1} M_{3}-0.06 M_{2} M_{3}\right. \\
& -0.02 m_{d_{3}}^{2}-0.32 m_{u_{3}}^{2}-0.29 m_{Q_{3}}^{2}+0.04 m_{H_{d}}^{2} \\
& \left.+0.70 m_{H_{u}}^{2}\right)\left.\right|_{M_{\mathrm{UV}}} .
\end{aligned}
$$

Here we have omitted terms with coefficients $<0.01$ (although they are internally kept in the following calculations). We have also

\footnotetext{
2 At the same order, the term $\bar{Y} Y /\left(M_{*}\right)^{2}$ can appear in the gauge kinetic functions, which slightly modifies the matching conditions for the gauge coupling constants Eq. (9). For $M_{*} \simeq 10^{17} \mathrm{GeV}$ and $\mathcal{O}$ (1) coefficients, however, the correction to the matching conditions is negligibly small.

3 Here, we have tacitly rescaled the spurion to absorb the typical size of the $\kappa$ in Eq. (6), so that the messenger scale is given by $M$.
}

neglected any terms involving the $A$-parameters at the messenger scale, since these are expected to be small in gauge mediation. The standard one-loop messenger gauge mediation expressions for the gaugino masses at $M_{\mathrm{UV}}=M$ are

$$
\begin{aligned}
M_{1} & =\frac{g_{1}^{2}}{16 \pi^{2}} \frac{F}{M}\left(\frac{3}{5} N_{2}+\frac{2}{5} N_{3}\right), \\
M_{2} & =\frac{g_{2}^{2}}{16 \pi^{2}} \frac{F}{M} N_{2}, \\
M_{3} & =\frac{g_{3}^{2}}{16 \pi^{2}} \frac{F}{M} N_{3},
\end{aligned}
$$

while the scalar soft masses are

$$
\begin{aligned}
m_{Q_{3}}^{2}= & 2\left(\frac{F}{M}\right)^{2}\left[\left(\frac{g_{3}^{2}}{16 \pi^{2}}\right)^{2} \cdot \frac{4}{3} N_{3}+\left(\frac{g_{2}^{2}}{16 \pi^{2}}\right)^{2} \cdot \frac{3}{4} N_{2}\right. \\
& \left.+\left(\frac{g_{1}^{2}}{16 \pi^{2}}\right)^{2} \cdot \frac{1}{60}\left(\frac{2}{5} N_{3}+\frac{3}{5} N_{2}\right)\right], \\
m_{u_{3}}^{2}= & 2\left(\frac{F}{M}\right)^{2}\left[\left(\frac{g_{3}^{2}}{16 \pi^{2}}\right)^{2} \cdot \frac{4}{3} N_{3}\right. \\
& \left.+\left(\frac{g_{1}^{2}}{16 \pi^{2}}\right)^{2} \cdot \frac{4}{15}\left(\frac{2}{5} N_{3}+\frac{3}{5} N_{2}\right)\right], \\
m_{d_{3}}^{2}= & 2\left(\frac{F}{M}\right)^{2}\left[\left(\frac{g_{3}^{2}}{16 \pi^{2}}\right)^{2} \cdot \frac{4}{3} N_{3}\right. \\
& \left.+\left(\frac{g_{1}^{2}}{16 \pi^{2}}\right)^{2} \cdot \frac{1}{15}\left(\frac{2}{5} N_{3}+\frac{3}{5} N_{2}\right)\right], \\
m_{H_{u}}^{2}= & m_{H_{d}}^{2}=2\left(\frac{F}{M}\right)^{2}\left[\left(\frac{g_{2}^{2}}{16 \pi^{2}}\right)^{2} \cdot \frac{3}{4} N_{2}\right. \\
& \left.+\left(\frac{g_{1}^{2}}{16 \pi^{2}}\right)^{2} \cdot \frac{3}{20}\left(\frac{2}{5} N_{3}+\frac{3}{5} N_{2}\right)\right] .
\end{aligned}
$$

Crucially, as mentioned before and as usual in minimal gauge mediation, the soft terms only depend on the ratio $F / M$ and on the messenger field content. In particular there is no dependence on the unknown Yukawa couplings $\kappa$ in Eqs. (5) or (6). ${ }^{4}$

Substituting Eqs. (12) and (13) into Eq. (11) we obtain

$$
\begin{aligned}
\left.m_{H_{u}}^{2}\right|_{M_{\mathrm{IR}}}= & \left(\frac{1}{16 \pi^{2}} \frac{F}{M}\right)^{2}\left(-0.215 N_{3}^{2}-0.449 N_{3}+0.044 N_{2}^{2}\right. \\
& \left.+0.150 N_{2}-0.015 N_{3} N_{2}\right)
\end{aligned}
$$

Suitable combinations for $\left(N_{2}, N_{3}\right)$ to ensure small negative $m_{H_{u}}^{2}$ at $M_{\mathrm{IR}}$ are given by $\left(N_{2}, N_{3}\right)=(17,7)$ or $\left(N_{2}, N_{3}\right)=(22,9)$. (Note that the ratio $N_{3}: N_{2}$ is always around $2: 5$ for a "gaugino focus point", for $N_{2}$ and $N_{3}$ sufficiently large. Here, both $7 / 17$ and $9 / 22$ are close to 0.41 .)

The one-loop running of the gauge couplings is shown in Fig. 1 for $\left(N_{2}, N_{3}\right)=(22,9)$ and in Fig. 2 for $\left(N_{2}, N_{3}\right)=(17,7)$. Above the GUT-breaking scale the couplings are seen to be quite large, and the reliability of the one-loop approximation should be questioned. We have therefore used two-loop running in this regime, in the (conservative) limit where any Yukawa couplings can be neglected.

\footnotetext{
4 Strictly speaking, the above soft masses are valid only for degenerate couplings $\kappa_{2, i}=\kappa_{3, a}$, whereupon all the messengers decouple at the same messenger scale. However, our results are not significantly altered even if the $\kappa$ are not all equal, as long as they are of a similar order of magnitude.
} 


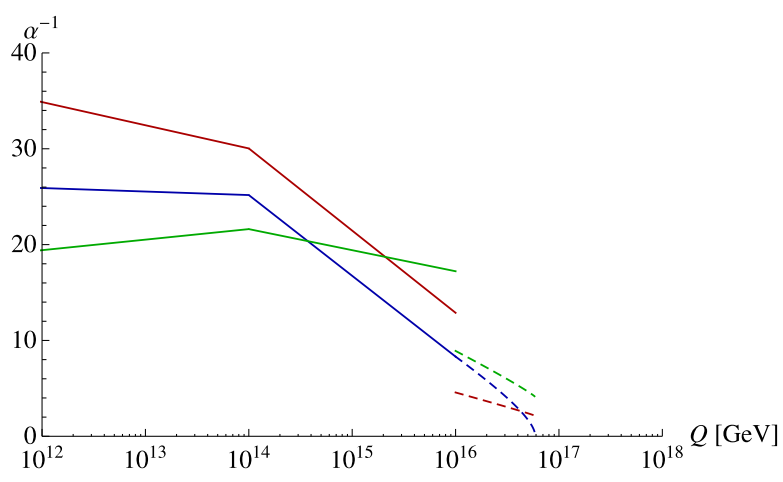

Fig. 1. Evolution of the gauge couplings for $\left(N_{2}, N_{3}\right)=(22,9)$. Solid red curve: $\alpha_{1}^{-1}$, solid blue curve: $\alpha_{2}^{-1}$, solid green curve: $\alpha_{3}^{-1}$. Dashed red curve: $\alpha_{1 \mathrm{H}}^{-1}$, dashed blue curve: $\alpha_{5}^{-1}$, dashed green curve: $\alpha_{3 \mathrm{H}}^{-1}$. Two-loop running is used above the matching scale $M_{\mathrm{GUT}}=10^{16} \mathrm{GeV}$, but the effect of Yukawa couplings is neglected. (For interpretation of the references to color in this figure legend, the reader is referred to the web version of this Letter.)

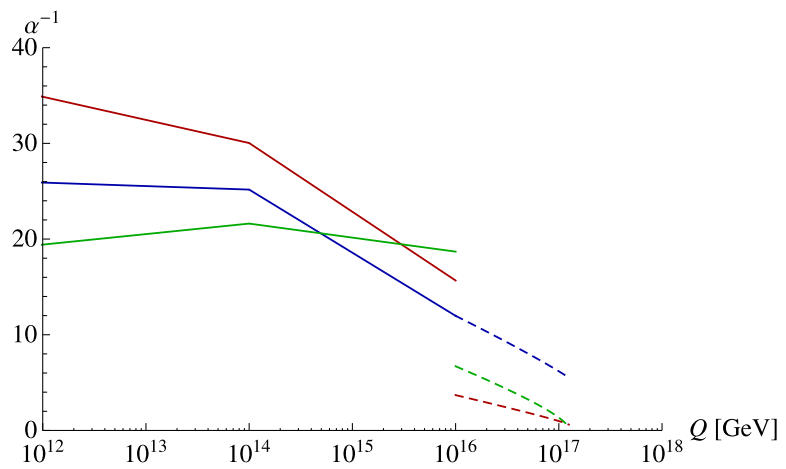

Fig. 2. Evolution of the gauge couplings for $\left(N_{2}, N_{3}\right)=(17,7)$. The colour code is the same as in Fig. 1. (For interpretation of the references to color in this figure legend, the reader is referred to the web version of this Letter.)

In the $\left(N_{2}, N_{3}\right)=(22,9)$ model, the two-loop correction is quite significant, and leads to the theory becoming perturbatively unreliable at scales below the previously assumed cutoff $M_{*}$. We therefore discard this possibility: the matter content of the model is too large to guarantee perturbative control an order of magnitude above the GUT-breaking scale. Note, however, that sizeable Yukawa couplings might still change this behaviour.

On the other hand, in the $\left(N_{2}, N_{3}\right)=(17,7)$ model a Landau pole is reached only around $M_{*}=10^{17} \mathrm{GeV}$. We therefore choose this model to compute a benchmark parameter point using SOFTSUSY [16]. Set $F=\left(4 \times 10^{9} \mathrm{GeV}\right)^{2}$, which implies that $\mu$ and $\sqrt{B_{\mu}}$ should be $\mathcal{O}\left(F / M_{*}\right) \sim 200 \mathrm{GeV}$. For practical purposes, they are fixed by requiring realistic electroweak symmetry breaking. In our benchmark point they come out slightly high: $\mu(M)=232 \mathrm{GeV}$ and $\sqrt{B_{\mu}}(M)=614 \mathrm{GeV}$. For scalar and gaugino soft masses, we use only the gauge-mediated contributions from Eqs. (12) and (13), disregarding possible "gravity-mediated" corrections $\mathcal{O}\left(F / M_{*}\right)$.

The low-energy sparticle spectrum is shown in Table 3. Note that the lightest Higgs mass is on the low end of what is compatible with the LHC observation, when taking a theory uncertainty of $3 \mathrm{GeV}$ into account. The reason is that, even with multi-TeV stops, a large Higgs mass is difficult to obtain if the A-terms are only generated radiatively. The superpartners are seen to be too heavy to be produced at the LHC as expected, with the exception of some relatively light higgsino-like charginos and neutralinos $\chi_{1}^{ \pm}, \chi_{1}^{0}$, and $\chi_{2}^{0}$. This is similar to previous models of high-scale gauge mediation in which the $\mu$ term is induced by subdominant gravity
Table 3

Mass spectrum for $\left(N_{2}, N_{3}\right)=(17,7)$, with $F=\left(4 \times 10^{9} \mathrm{GeV}\right)^{2}, M=10^{14} \mathrm{GeV}, \mu=$ $230 \mathrm{GeV}$, and $\sqrt{B_{\mu}}=614 \mathrm{GeV}$. For these parameters, $\tan \beta=49$.

\begin{tabular}{|c|c|c|c|}
\hline Particle & Mass [GeV] & Particle & Mass [GeV] \\
\hline$h_{0}$ & 123 & $A_{0}$ & 3000 \\
\hline$\chi_{1}^{0}$ & 207 & $H^{ \pm}$ & 3000 \\
\hline$\chi_{1}^{ \pm}$ & 208 & $\tilde{g}$ & 7000 \\
\hline$\chi_{2}^{0}$ & 209 & $\tilde{\tau}_{1}$ & 2000 \\
\hline$\chi_{3}^{0}$ & 2900 & other sleptons & $3200-6000$ \\
\hline$\chi_{4}^{0}$ & 6900 & $\tilde{t}_{1}$ & 5000 \\
\hline$\chi_{2}^{ \pm}$ & 6900 & $\tilde{t}_{2}$ & 7500 \\
\hline$H_{0}$ & 3000 & other squarks & $6400-8600$ \\
\hline
\end{tabular}

mediation effects, see e.g. [4]. Light higgsinos will be difficult to see at the LHC, but could be discovered at a future linear collider.

To estimate the fine-tuning, we take the usual definition

$\Delta=\max _{\text {parameters } a} \frac{\partial \log m_{Z}}{\partial \log a}, \quad$ fine-tuning $=\frac{1}{\Delta}$.

There are only two independent dimensionful parameters $a$ which enter $m_{Z}$ (at large $\tan \beta$ ). Namely,

$m_{Z}^{2}=-\left.2\left(|\mu|^{2}+m_{H_{u}}^{2}\right)\right|_{M_{\mathrm{IR}}}=0.42 m_{\mathrm{GMSB}}^{2}-\left.1.62|\mu|^{2}\right|_{M_{\mathrm{UV}}}$

where

$m_{\mathrm{GMSB}} \equiv \frac{1}{16 \pi^{2}} \frac{F}{M} \approx 1 \mathrm{TeV}$.

While this relation is not precise enough to predict $m_{Z}$ accurately, it does serve to illustrate that the residual fine-tuning is of the order $\left(m_{Z} / m_{\mathrm{GMSB}}\right)^{2}$ (because the coefficient of $m_{\mathrm{GMSB}}$ is $\left.\mathcal{O}(1)\right)$. For the present benchmark point, this implies that $1 / \Delta \sim \mathcal{O}(1 \%)$, which is a considerable improvement over generic models with similarly heavy superpartners. It should be pointed out, however, that the sensitivity of the focus point cancellation to the dimensionless Standard Model couplings (which are usually not included into the fine-tuning definition) is likely very high.

One might object that the particular choice of messenger content in our model constitutes a fine-tuning by itself which we should take into account. Our take is that the field content of a given model should be regarded as part of the model definition, and therefore not included in the fine-tuning computation. (Note also that the canonical quantitative measure of fine-tuning, the sensitivity of the weak scale with respect to parameter variations, is ill-defined for "discrete parameters" such as the number of particle flavours.) While it is meaningful to compare different models, defined by different symmetries and field content, with regards to their fine-tuning in their respective continuous parameters, there is no natural measure which a priori prefers any particular model over another. At best, we can aim for simplicity and minimality. By these standards our peculiar messenger field content may admittedly be considered a drawback of our model-as often in model-building, reduced fine-tuning comes at the price of a more elaborate construction. However, the fairly large messenger numbers in our model are also a consequence of demanding that the gauge couplings should be perturbative above the GUT-breaking scale. This condition thus serves as an independent motivation for considering a model in which $N_{2}$ and $N_{3}$ are of the order 10-20.

Finally, if the $Z$ superfield of the previous section is identified with the goldstino multiplet, then the LSP is a $4 \mathrm{GeV}$ gravitino. If furthermore $R$-parity is conserved, within standard cosmology, the decays of the $\chi_{1}^{0}$ NLSP will spoil the successful prediction of light element abundances during Big Bang Nucleosynthesis. In the current model, however, the relic abundance of the NSLP is rather 
low, $\Omega_{\chi} h^{2} \lesssim 10^{-2}$, due to the large annihilation cross section of the Higgsino. Therefore the BBN problem can solved by slightly lowering the SUSY breaking, such as to lower the gravitino mass to $\lesssim 1 \mathrm{GeV}$ which reduces the higgsino lifetime to $\mathcal{O}\left(10^{2}\right)$ seconds $[17,18] . .^{5}$ On the other hand, the actual goldstino direction of the hidden sector may have other components besides $Z$ (as in the model of the following section), in which case the gravitino could also be heavier than $\chi_{1}^{0}{ }^{6}$

\section{The origin of the SUSY breaking spurion}

So far, we have simply assumed that the messengers couple to a SUSY-breaking spurion field $Z$ whose vacuum expectation value is

$\langle Z\rangle=M+F \theta^{2}$.

In this section, we sketch a possible dynamical origin of $F$ and $M$. We consider the model of cascade SUSY breaking which was developed in Refs. $[19,20]$. This model contains a primary SUSY-breaking field $Z_{0}$ and a secondary SUSY-breaking field $Z_{1}$, the latter of which will be identified with $Z$.

In the cascade SUSY breaking model, the Kähler potential and superpotential are

$K=Z_{0}^{\dagger} Z_{0}+Z_{1}^{\dagger} Z_{1}-\frac{c_{0}^{2}}{4 \Lambda^{2}}\left(Z_{0}^{\dagger} Z_{0}\right)^{2}+\frac{c_{1}^{2}}{\Lambda^{2}} Z_{0}^{\dagger} Z_{0} Z_{1}^{\dagger} Z_{1}+\cdots$,

$W=\Lambda^{2} Z_{0}+\frac{h}{3} Z_{1}^{3}+\kappa Z_{1} \Phi \Phi$,

where $\Lambda$ denotes a dimensionful parameter while $c_{0,1}, h$ and $\kappa$ are dimensionless coefficients. In the following, we take $h$ and $\kappa$ to be real and positive by rotating the phases of the fields appropriately. We are assuming that the higher dimensional Kähler potential terms are generated radiatively by integrating out certain fields in a generalized O'Raifeartaigh model $[19,20]$. It can be shown that $c_{0}^{2}>0$ when the quartic $Z_{0}$ term is perturbatively generated by integrating out fields with $R$-charges 0 or 2 [21]. Similarly, for $c_{1}^{2}>0$ one needs $Z_{1}$ to couple to fields with $R$-charges other than 0 or 2 $[19,20]$.

In this model, there is an $R$-symmetric but SUSY-breaking vacuum at

$Z_{0}=0$,

$F_{Z_{0}}=\Lambda^{2}$

around which the primary SUSY-breaking field obtains a mass

$m_{Z_{0}}^{2}=c_{0}^{2} \Lambda^{2}$.

Once $Z_{0}$ breaks SUSY, the secondary SUSY-breaking field $Z_{1}$ obtains a soft SUSY-breaking mass term. The scalar potential for $Z_{1}$ is given by

$V\left(Z_{1}\right) \simeq-m_{Z_{1}}^{2}\left|Z_{1}\right|^{2}+\left|h Z_{1}^{2}\right|^{2}, \quad m_{Z_{1}}^{2}=c_{1}^{2} \frac{\left|F_{Z_{0}}\right|^{2}}{\Lambda^{2}}=c_{1}^{2} \Lambda^{2}$.

Therefore, for $m_{Z_{1}}^{2}>0$, the secondary SUSY-breaking field obtains a non-vanishing expectation value,

\footnotetext{
5 Note however that the gravitino cannot be made arbitrarily light. To obtain an MSSM spectrum similar to the one of Table 3, the messenger scale also would need to be lowered accordingly, keeping $F / M$ constant. But a too low messenger scale would result in a too large $g_{5}$ coupling at $M_{\mathrm{GUT}}$, which would then again blow up very quickly.

6 In the Higgsino LSP case, the thermal relic abundance is too small to account for the observed dark matter density, so one would need non-thermal sources for the Higgsino or another dark matter candidate.
}

$\left\langle Z_{1}\right\rangle \simeq \frac{m_{Z_{1}}}{\sqrt{2} h} \simeq \frac{c_{1}}{\sqrt{2} h} \Lambda$

which breaks SUSY by

$F_{Z_{1}}=h\left\langle Z_{1}^{*}\right\rangle^{2} \simeq \frac{m_{Z_{1}}^{2}}{2 h}=\frac{c_{1}^{2}}{2 h} \Lambda^{2}$.

In this way, secondary SUSY breaking is initiated by spontaneous $R$-symmetry breaking which is, in turn, triggered by fundamental SUSY breaking.

Through the coupling between the messengers and $Z_{1}$ in Eq. (19), the secondary SUSY breaking field $Z_{1}$ plays the role of the spurion in the previous sections, i.e.

$M=\kappa\left\langle Z_{1}\right\rangle \simeq \frac{\kappa c_{1}}{\sqrt{2} h} \Lambda, \quad F=\kappa F_{Z_{1}} \simeq \frac{\kappa c_{1}^{2}}{2 h} \Lambda^{2}$.

In the following, we assume that $c_{1}^{2} \simeq 2 h$ and $\kappa \simeq 1$, in order to obtain a gravitino mass which is as low as possible for a given $F$, i.e. $F \simeq F_{Z_{1}} \simeq F_{Z_{0}}$, while keeping the messenger mass as high as possible. ${ }^{7}$ Under these assumptions, the parameters used in the previous section,

$M \simeq 10^{14} \mathrm{GeV}, \quad F \simeq\left(4 \times 10^{9} \mathrm{GeV}\right)^{2}$,

are obtained by choosing

$\Lambda \simeq 4 \times 10^{9} \mathrm{GeV}, \quad c_{1} \simeq 5 \times 10^{-5}$.

Finally, let us comment on the origins of the $\mu$ and $B_{\mu}$ term. As mentioned earlier, one may consider "gravity-mediated" contributions,

$K=c_{H} \frac{Z^{\dagger}}{M_{*}} H_{u} H_{d}+c_{B} \frac{Z^{\dagger} Z}{M_{*}^{2}} H_{u} H_{d}+$ h.c.

which leads to

$\mu=\sqrt{3} c_{H} \frac{M_{\text {Planck }}}{M_{*}} m_{3 / 2}$,

$\sqrt{B_{\mu}}=\sqrt{3} c_{B}^{1 / 2} \frac{M_{\text {Planck }}}{M_{*}} m_{3 / 2}$.

Thus, with the coefficients $c_{H}$ and $c_{B}$ of order unity and $M_{*} \simeq$ $10^{17} \mathrm{GeV}$, one obtains values for $\mu$ and $B_{\mu}$ of the order of the weak scale as desired.

It should be noted that one of the phases of $c_{H, B}$ cannot be eliminated by field redefinitions, which leads to $\mathrm{CP}$-violating processes and gives rise to, for instance, an electron electric dipole moment (EDM). In fact, if the relative phase between $\mu$ and $B_{\mu}$ is of order unity, the predicted electron EDM slightly exceeds the current limit for the mass spectrum in Table 3. Avoiding the bound requires some amount of tuning between $c_{H}$ and $c_{B}$.

Similarly, completely generic gravity-mediated contributions to soft masses and $A$-terms would lead to unacceptably large flavour changing neutral currents. While the soft terms are dominated by the flavour-universal gauge-mediated contributions, subdominant flavour-violating corrections are still potentially dangerous, see e.g. [22,23]. Keeping them under control also requires some tuning or an underlying symmetry. However, a detailed analysis of the flavour and CP problems in our model is beyond the scope of this work.

\footnotetext{
7 As mentioned above, another possibility would be to have a much heavier gravitino mass and a Higgsino LSP. In this case, parameter choices such as $c_{1} \ll h$ and $\kappa \ll 1$ are also allowed.
} 


\section{Conclusions}

We have shown that focus point gauge mediation can naturally be embedded into a model of $\mathrm{SU}(5) \times \mathrm{U}(3)_{\mathrm{H}}$ product group unification. For the cutoff of the product group unification model to be substantially higher than the GUT-breaking scale, we have added to the MSSM a number of colour triplet and weak doublet pairs at intermediate energies. In terms of full $\mathrm{SU}(5) \times \mathrm{U}(3)_{\mathrm{H}}$ representations, this corresponds to adding several $\mathbf{5} \oplus \overline{\mathbf{5}}$ and $\mathbf{3} \oplus \overline{\mathbf{3}}$ pairs. Importantly, if the cutoff of the model is to be significantly beyond the GUT-breaking scale, this implies that the number of extra states should be fairly large. For instance, with $N_{2}=17$ extra doublet pairs and $N_{3}=7$ extra triplet pairs, the cutoff can be postponed until $M_{*} \approx 10^{17} \mathrm{GeV}$. If the number of extra states is further increased (as in the $\left(N_{2}, N_{3}\right)=(9,22)$ model which we also discussed), a Landau pole in the SU(5) gauge coupling appears just above the GUT-breaking scale. Too few extra states would also imply a lower cutoff scale, as can be seen from the extreme case $\left(N_{2}, N_{3}\right)=(0,0)$ where the $\mathrm{U}(1)_{\mathrm{H}}$ gauge coupling would have to be non-perturbative already at $M_{\mathrm{GUT}}$.

The observation that both $\mathrm{U}(3)_{\mathrm{H}}$ couplings become strong at $M_{*} \approx 10^{17} \mathrm{GeV}$ may indicate that the $\mathrm{U}(3)_{\mathrm{H}}$ gauge bosons are composite states at the cutoff scale $M_{*}$ [24-26]. To realize a compositeness scale between the GUT scale and the Planck scale, introducing additional charged states, as we have done, in fact becomes a necessity. The SU(5) gauge bosons, on the other hand, may be elementary (or they might be composite as well, if further $\mathbf{5} \oplus \overline{\mathbf{5}}$ multiplets with GUT-scale masses are introduced).

The extra weak doublets and colour triplets act as gauge mediation messengers, leading to the non-unified soft term mass ratios which are required for a focus point-like cancellation between the radiative corrections to the Higgs potential, and consequently a little hierarchy between the soft mass scale and the electroweak scale. The superpartners, except for some higgsino-like neutralinos and charginos, are predicted to be very heavy and out of LHC reach. The resulting Higgs mass is compatible with $125 \mathrm{GeV}$, while the fine-tuning is still comparatively modest.

\section{Acknowledgements}

This work is supported by Grant-in-Aid for Scientific research from the Ministry of Education, Science, Sports, and Culture (MEXT), Japan, No. 22244021 (T.T.Y.), No. 24740151 (M.I.), and also by the World Premier International Research Center Initiative (WPI Initiative), MEXT, Japan.

\section{References}

[1] H. Abe, T. Kobayashi, Y. Omura, Phys. Rev. D 76 (2007) 015002, arXiv:hepph/0703044.

[2] D. Horton, G.G. Ross, Nucl. Phys. B 830 (2010) 221, arXiv:0908.0857 [hep-ph].

[3] F. Brümmer, W. Buchmüller, J. High Energy Phys. 1205 (2012) 006, arXiv:1201.4338 [hep-ph].

[4] F. Brümmer, W. Buchmüller, J. High Energy Phys. 1107 (2011) 010, arXiv:1105.0802 [hep-ph]

[5] J.E. Younkin, S.P. Martin, Phys. Rev. D 85 (2012) 055028, arXiv:1201.2989 [hep$\mathrm{ph}]$.

[6] S. Antusch, L. Calibbi, V. Maurer, M. Monaco, M. Spinrath, arXiv:1207.7236 [hep-ph].

[7] T.T. Yanagida, N. Yokozaki, arXiv:1301.1137 [hep-ph].

[8] K.L. Chan, U. Chattopadhyay, P. Nath, Phys. Rev. D 58 (1998) 096004, arXiv:hep$\mathrm{ph} / 9710473$.

[9] J.L. Feng, K.T. Matchev, T. Moroi, Phys. Rev. Lett. 84 (2000) 2322, arXiv:hep$\mathrm{ph} / 9908309$.

[10] J.L. Feng, K.T. Matchev, T. Moroi, Phys. Rev. D 61 (2000) 075005, arXiv:hepph/9909334.

[11] T. Yanagida, Phys. Lett. B 344 (1995) 211, arXiv:hep-ph/9409329.

[12] J. Hisano, T. Yanagida, Mod. Phys. Lett. A 10 (1995) 3097, arXiv:hep$\mathrm{ph} / 9510277$.

[13] K.I. Izawa, T. Yanagida, Prog. Theor. Phys. 97 (1997) 913, arXiv:hep-ph/9703350.

[14] M. Ibe, Y. Shinbara, T.T. Yanagida, Phys. Lett. B 639 (2006) 534, arXiv:hep$\mathrm{ph} / 0605252$.

[15] K. Nakayama, F. Takahashi, T.T. Yanagida, Phys. Rev. D 86 (2012) 043507, arXiv:1112.0418 [hep-ph].

[16] B.C. Allanach, Comput. Phys. Commun. 143 (2002) 305, arXiv:hep-ph/0104145.

[17] M. Kawasaki, K. Kohri, T. Moroi, Phys. Rev. D 71 (2005) 083502, arXiv:astro$\mathrm{ph} / 0408426$.

[18] K. Jedamzik, Phys. Rev. D 74 (2006) 103509, arXiv:hep-ph/0604251.

[19] M. Ibe, Y. Shirman, T.T. Yanagida, J. High Energy Phys. 1012 (2010) 027, arXiv:1009.2818 [hep-ph].

[20] J.L. Evans, M. Ibe, M. Sudano, T.T. Yanagida, J. High Energy Phys. 1203 (2012) 004, arXiv:1103.4549 [hep-ph].

[21] D. Shih, J. High Energy Phys. 0802 (2008) 091, arXiv:hep-th/0703196.

[22] G. Hiller, Y. Hochberg, Y. Nir, J. High Energy Phys. 0903 (2009) 115, arXiv:0812.0511 [hep-ph].

[23] G. Hiller, Y. Hochberg, Y. Nir, J. High Energy Phys. 1003 (2010) 079, arXiv:1001.1513 [hep-ph].

[24] L.D. Landau, A.A. Abrikosov, I.M. Khalatnikov, Dokl. Akad. Nauk USSR 95 (1954) 773,1177 ;

L.D. Landau, A.A. Abrikosov, I.M. Khalatnikov, Dokl. Akad. Nauk USSR 96 (1954) 261.

[25] L.D. Landau, I.Y. Pomeranchuk, Dokl. Akad. Nauk Ser. Fiz. 102 (1955) 489.

[26] L.D. Landau, in: W. Pauli (Ed.), Niels Bohr and the Development of Physics, Pergamon Press, 1955. 